$62^{\text {ème }}$ Congrès de la SFCO, 02013 (2014)

DOI: $10.1051 /$ sfco/20146202013

(C) Owned by the authors, published by EDP Sciences, 2014

\title{
Evaluation in vitro de l'effet d'une fatigue dynamique sur la fuite des connectiques implantaires par la méthode de diffusion gazeuse, sur huit types d'implants.
}

\author{
Fauroux MA', ${ }^{1,2}$ Anxionnat $C^{1}$, Fistes $\mathbf{M}^{1}$, Guillot JM', Torres $\mathrm{JH}^{1,2}$ \\ 1 Service d'Odontologie du CHRU, 545 avenue Pr JL Viala, 34295, Montpellier cedex 5, FRANCE \\ 2 EA4203, UFR d'Odontologie, 454 avenue Pr JL Viala, 34193, Montpellier cedex 5, FRANCE
}

La jonction entre le pilier prothétique et l'implant dans les systèmes implantaires en deux parties représente une zone potentielle de contamination et de prolifération bactérienne. L'importance du hiatus qui existe entre ces deux pièces peut être estimée in vitro en mesurant le passage d'un liquide ou d'un gaz à travers cet assemblage. La méthode par diffusion gazeuse, utilisée lors d'études antérieures, a déjà fait la preuve de son efficacité (Torres et al. 2011). Une première étude sur treize types d'implants a mis en évidence des différences de fuite lorsque les piliers prothétiques étaient vissés aux implants, aux forces de serrage recommandées par les sociétés commerciales (Fauroux et al. 2014). Pour se rapprocher des conditions réelles de mastication, l'étude présentée ici avait pour but d'évaluer l'effet théorique d'une contrainte répétée (stress mécanique) sur la fuite des connectiques implantaires pour huit systèmes.

Cette étude devait répondre à trois questions. La première consistait à mettre en évidence des différences de la fuite parmi les huit types d'implants (pris deux par deux) après application d'une contrainte répétée. Ensuite, d'évaluer l'effet général du stress sur l'ensemble de tous les implants quelle que soit la marque et le type d'implants. Et enfin, de comparer la fuite des connectiques avant et après stress pour chacun des huit types d'implants. Les systèmes concernés par ces tests ont été : Euroteknika (Aesthetica ${ }^{\circledR}$; Natea ${ }^{\circledR}$; Universal $+{ }^{\circledR}$ ) ; Tekka (In-Kone Universal $\left.{ }^{\circledR}\right)$; Straumann $\left(\right.$ Bone Level $\left.^{\circledR}\right)$; Nobel Biocare $\left(\right.$ Mk III TiU $\left.^{\circledR}\right)$; Astratech (Osseospeed $\left.{ }^{\circledR}\right)$; Dentsply (Ankylos C/X ${ }^{\circledR}$ ). Les tests ont été effectués sur 5 implants pour chacun des systèmes (soit 40 implants au total). La contrainte a été appliquée 2 millions de fois sur une coiffe sphérique portée par le pilier prothétique, avec un angle de 30 degrés par rapport au grand axe du pilier, selon la norme NF EN ISO 14801.

Les résultats de cette étude ont montré l'existence d'un effet général de la contrainte sur la fuite, ainsi que des différences de fuite entre certains systèmes après contrainte. Les systèmes Nobel Biocare Mk III TiU ${ }^{\circledR}$, Astratech Osseospeed ${ }^{\circledR}$, Dentsply Ankylos $\mathrm{C} / \mathrm{X}^{\circledR}$; Euroteknika Aesthetica ${ }^{\circledR}$ et Universal ${ }^{\circledR}$ ont diminué leur fuite alors que les autres systèmes ont majoré ou stabilisé leur fuite avant et après stress.

En conclusion, ces observations relativisent l'extrapolation clinique des résultats obtenus in vitro en l'absence de stress mécanique. Lorsqu'il s'agit de tester l'étanchéité des connexions, des essais de fatigue dynamique devraient donc être entrepris de manière systématique, malgré le temps que demandent ces tests (plusieurs dizaines d'heures pour chaque échantillon). Afin de compléter l'étude d'une relation entre application d'une contrainte répétée et fuite de la connectique implantaire, des coupes transversales de ces connectiques doivent à présent être observées au microscope électronique à balayage. Une inhomogénéité du hiatus, corrélée à l'orientation de l'application de la contrainte, pourrait permettre d'objectiver cet effet.

FAUROUX Marie-Alix marie-alix.fauroux@univ-montp1.fr

This is an Open Access article distributed under the terms of the Creative Commons Attribution License 4.0, which permits unrestricted use, distribution, and reproduction in any medium, provided the original work is properly cited. 